\title{
A hybrid method for traumatic brain injury lesion segmentation
}

\author{
Ahmad Yahya Dawod ${ }^{1}$, Aniwat Phaphuangwittayakul ${ }^{1}$, Salita Angkurawaranon ${ }^{2}$ \\ ${ }^{1}$ International College of Digital Innovation, Chiang Mai University, Chiang Mai, Thailand \\ ${ }^{2}$ Faculty of Medicine, Chiang Mai University, Chiang Mai, Thailand
}

\begin{tabular}{l} 
Article Info \\
\hline Article history: \\
Received Mar 24, 2021 \\
Revised Aug 13, 2021 \\
Accepted Sep 4, 2021 \\
\hline Keywords: \\
Edge detection \\
Free-form object model \\
Hybrid method \\
Image segmentation \\
Simple linear iterative \\
clustering algorithm
\end{tabular}

\section{Article history:}

Received Mar 24, 2021

Revised Aug 13, 2021

Accepted Sep 4, 2021

\section{Keywords:}

Free-form object model

Hybrid method

Simple linear iterative

clustering algorithm

\begin{abstract}
Traumatic brain injuries are significant effects of disability and loss of life. Physicians employ computed tomography (CT) images to observe the trauma and measure its severity for diagnosis and treatment. Due to the overlap of hemorrhage and normal brain tissues, segmentation methods sometimes lead to false results. The study is more challenging to unitize the AI field to collect brain hemorrhage by involving patient datasets employing CT scans images. We propose a novel technique free-form object model for brain injury CT image segmentation based on superpixel image processing that uses CT to analyzing brain injuries, quite challenging to create a high outstanding simple linear iterative clustering (SLIC) method. The method maintains a strategic distance of the segmentation image to reduced intensity boundaries. The segmentation image contains marked red hemorrhage to modify the free-form object model. The contour labelled by the red mark is the output from our free-form object model. We proposed a hybrid image segmentation approach based on the combined edge detection and dilation technique features. The approach diminishes computational costs, and the method accomplished $94.84 \%$ accuracy. The segmenting brain hemorrhage images are achieved in the clustered region to construct a free-form object model. The study also presents further directions on future research in this domain.
\end{abstract}

This is an open access article under the CC BY-SA license.

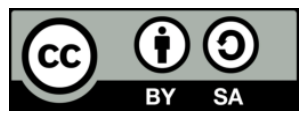

\section{Corresponding Author:}

Ahmad Yahya Dawod

International College of Digital Innovation, Chiang Mai University

239 Nimmanahaeminda Road, Suthep, Muang, Chiang Mai 50200, Thailand

Email: ahmadyahyadawod.a@cmu.ac.th

\section{INTRODUCTION}

Traumatic brain injuries (TBI) are generally affected by external forces on the brain in accidents or head strikes and considered as significant cause of disabilities and death. TBI can range from an intracerebral hemorrhage, subdural hematoma, epidural hematoma, cerebral contusion, and cerebellar hemorrhage. Medical imaging modalities such as computed tomography (CT) scans are wide to establish severity and diagnosis and decide targeted therapy within early hours of injuries. An accurate and appropriate decision is necessary for physicians to manage the patient more precisely and ensure better diagnosis and outcomes. The segmentation of images is one of the most essential image processing and computer vision procedures. Picture segmentation is the way an image is separated into segments. It is most helpful for image detection purposes for these applications; it is inefficient to process the whole image. Segmentation of the image is utilized to segment from the image for further processing. Image segmentation techniques in computer vision partition the image into several parts based on specific image features like pixel intensity value, color, and texture [1]. Numerous approaches for brain image segmentation [2] have been proposed. Around these approaches work on the values of the clustering model and intensity threshold procedures. However, this conventional segmentation fails to classify subdural successfully as they are hard to define by a specific 
model, and subdural pose a varied range of concentration for various patients. The segmentation image could be a preparation for apportioning the image into sets of pixels [3]. Feature extractions and inquiries on medical image processing were always challenging, especially in pixel-based approaches. The most significant disadvantage of region-based techniques and histogram methods is their lack of adherence to flexibility, compactness, and boundaries. So, during segmentation, the region of interest's edges and border become very blurry [4]. A technique clustering pixels into standardized image areas is superpixels (SP). Then, the use of SPs has developed significantly in various image processing applications. Meanwhile, superpixels regions possess similar texture characteristics and color. Tasli et al. [5] offers an efficient illustration of the image. This property underpins the supposition that pixels in the same superpixel fit to a similar semantic object [6]. Stemming from this idea, all pixels in an SP can be allocated to explicit models representing segmentation constructions [7] is low in memory efficiency and high computational cost. Hence the super-pixel-based segmentation approach using simple linear iterative clustering (SLIC) was adopted and tested by various researchers recently and proved to be efficient and superior to the classical pixel-based approaches edge-preservation. To the best of the researcher's knowledge, the SLIC algorithm was able to segment the 3D images of brain hemorrhages. Hence, this research is novel in using the SLIC algorithm to segment brain hemorrhages [8]. We suggest a new technique that employs a free-form shape of a hemorrhage model that suits any injury type. In this paper, we proposed the eight steps approach of i) image acquisition: converting digital imaging and communications in medicine (DICOM) images to jpeg format; ii) preprocessing: linear transformation and windowing of the images; iii) image processing: use SLIC algorithm for creating superpixels; iv) edge detection: eliminate false boundaries; v) region growing; vi) dilation to clean the image from the noise; vii) a hybrid method; viii) segmentation region to get accurate: segmentation was assessed with 30,000 brain hemorrhage images obtained from Maharaj Nakorn Chiang Mai Hospital, Thailand.

\section{RESEARCH METHOD}

This section describes our new technique as an eight-stage by using a novel free-from object model. The approach for improving the accuracy and efficiency of automated 3D image processing of CT in analyzing brain injuries is depicted in Figure 1.

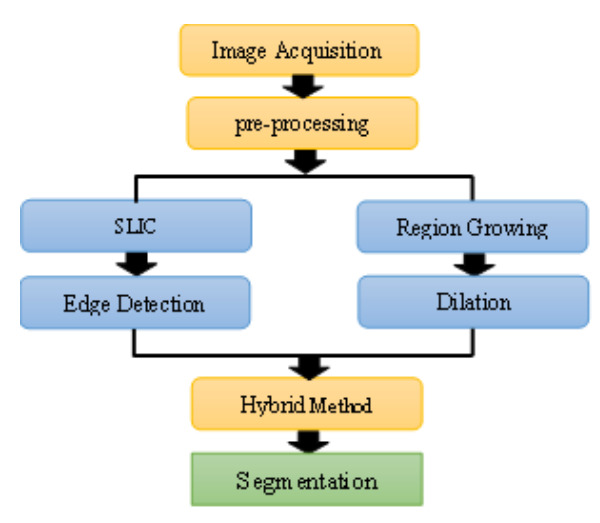

Figure 1. Illustration of the proposed method

\subsection{Image acquisition}

In the first step of the input image, we converted more than 30,000 3D DICOM format brain images into 2D JPEG format with a spatial resolution of $512 \times 512$; slice thickness of $1 \mathrm{~mm}$ to $1.5 \mathrm{~mm}$, and distance between each slice of $0.5 \mathrm{~mm}$. An illustrative example of sample CT brain slices after the pre-processing step of converting from 3D to 2D is presented in Figure 2. The slices shown in the image are linearly transformed images after intensity windowing. The examples of two TBI subtypes including epidural hematoma (EDH) and subdural hematoma (SDH) are illustrated in Figures 2(a) and (b), respectively. The researchers for segmentation processing used IDE Python Spyder.

\subsection{Pre-processing}

Pre-processing is the most significant step in the introduced method. The first step of pre-processing the removal of skull and CT scans filtering can reduce the data before the segmentation technique. To enhance 
the automatic method based on clustering, Morphological processing, and edge detection was employed to perform the removal skull [9]. We linearly transformed the input image intensities using intensity windowing and contrast adjustment techniques [10]. The desired window intensity was adopted from the provided DICOM header information. The image's intensities are first rescaled to a range between the high-intensity region (HIR) value and the low-intensity region (LIR) value. We are pre-processing using the Plot image intensity histogram provided between (80-200) the upper range, as shown in Figure 3.

From brain CT hemorrhage image intensity histogram then, to find the first local minima of each divided region (five regions in the histogram) show in Figure 4, the values of these minima are selected as the threshold for the image segmentation process. We obtained three significant points calculate the first point one is a threshold for background or black pixels after Point four is a threshold for hemorrhage finally; five points is a threshold for the skull of white pixels shows in Figure 5. We mention a grayscale value between range 0-255 with different intensity low or high in terms of tissue represented by the wave of the upper and lower threshold.

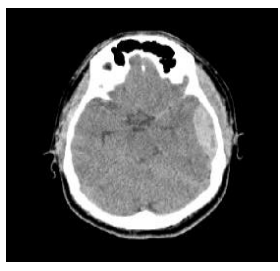

(a)

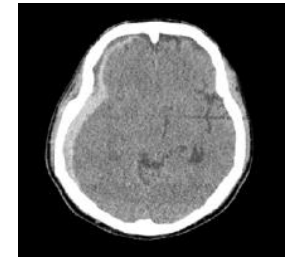

(b)

Figure 2. The head with the skull: (a) EDH injury and (b) SDH injury

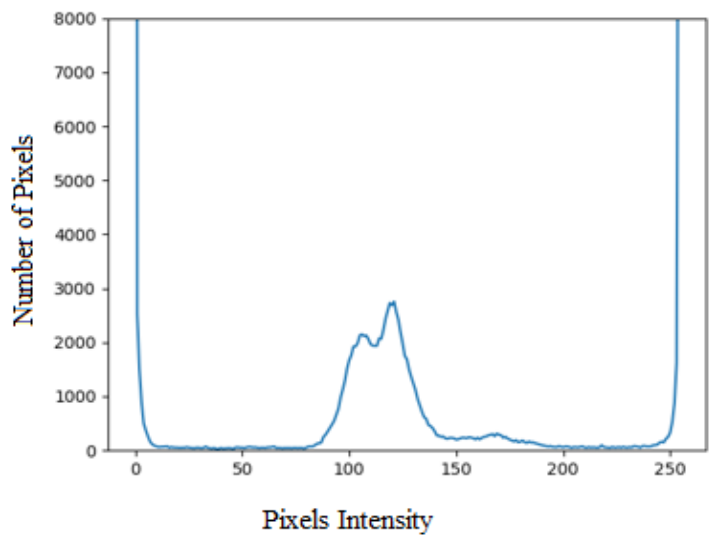

Figure 3. The plot of the intensity histogram

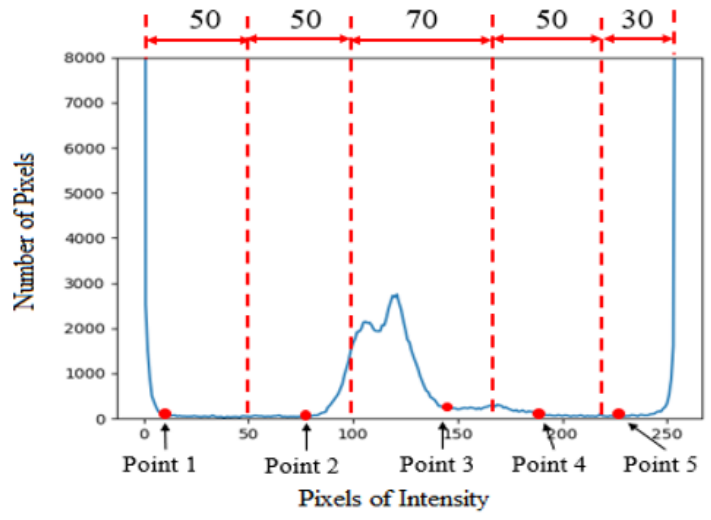

Figure 4. Shows the first local minima by five points

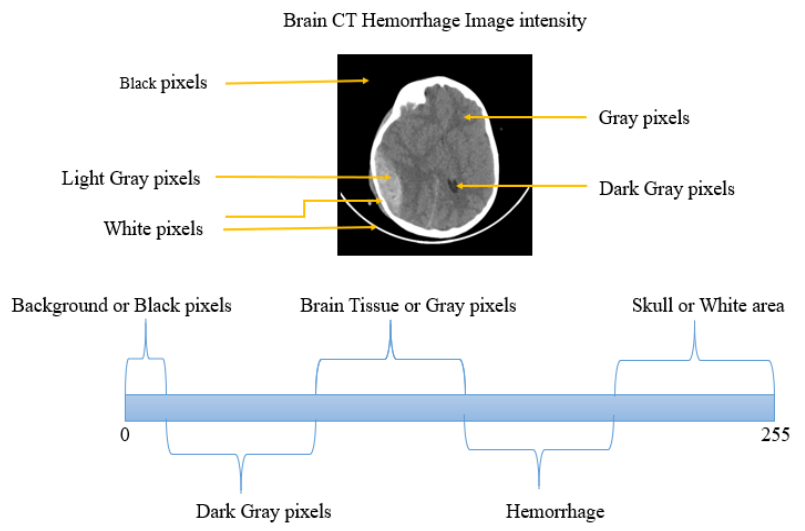

Figure 5. Brain CT hemorrhage image intensity 
In terms of using point five as the threshold to filter the only skull, partly used binary image shown in Figure 6(a) then find contours and select the most massive area show Figure 6(b). After that, the brain region without noise is cropped as shown in Figure 6(c). We then filter the skull out and keep only the inside brain region as illustrated in Figure 6(d).

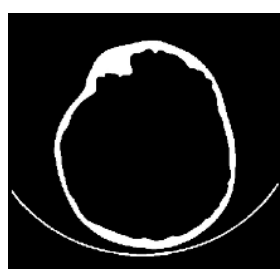

(a)

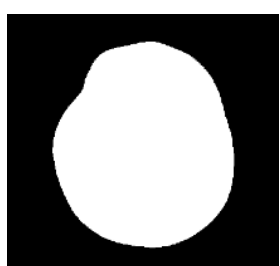

(b)

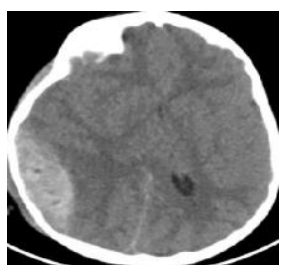

(c)

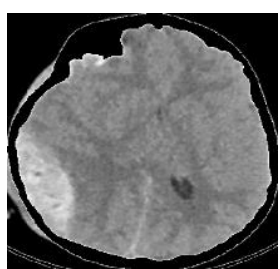

(d)

Figure 6. Different processing for proposal method: (a) The larger region of the brain, (b) cropping the brain removed, (c) the grayscale technique for the brain, and (d) removal of the skull

The (1) for the linear transformation of images as follows:

$$
H U=\left(P V * R_{S}\right)+R_{1}
$$

where HU, PV, Rs, and Ri stand for Hounsfield unit, raw pixel value, rescale gradient, and rescale intercept, respectively. The region of interest within the hemorrhaging blood has higher HU values than the soft tissues and cerebral spinal fluid (CSF), as seen in Figure 7. Figure 7(a) presents the samples of brain region without noise outside skull. Figure 7(b) shows the samples of brain in specific range of HU. We converted the Hounsfield units scale image to grayscale by setting window width $=151$, window level $=114$. The Hounsfield values are in the range of 50-95 to coincide with the Hounsfield values of brain hemorrhages.
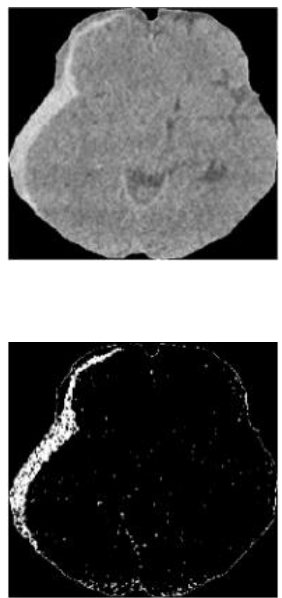

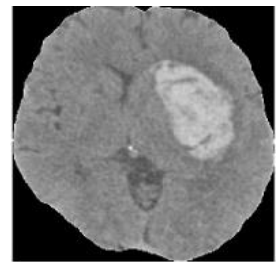

(a)

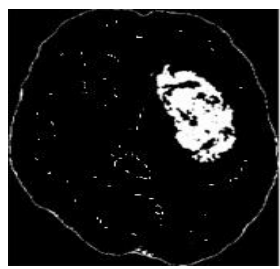

(b)
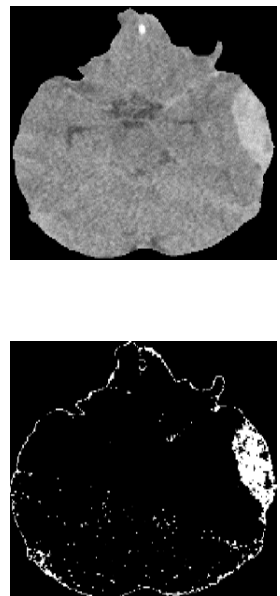

Figure 7. Convert Hounsfield units scale image: (a) the brain without a skull and (b) grayscale with noise

\subsection{Simple linear iterative clustering (SLIC)}

We apply the SLIC technique for segmentation introduced in [11]. Each slice is gridded to small equal square sections of a user-defined size, considered initial superpixels. To perform the process, we need the centers of each region for further calculations. In the first instance, the geometrical centers of these squares are considered as superpixel region centers. Then, the center locations are simplified according to the mean value of each cluster pixel coordinates. Distance between each pixel in the image to the boundary cluster centers are computed, and a label of the closest cluster center is assigned to the target pixel. If $a$ and $b$ are considered as pixel location coordinates values with user-specified grid size (s), the gray space distance $\left(d_{c}\right)$, the Euclidean distance $\left(d_{s}\right)$ between the $\mathrm{j}^{\text {th }}$ pixel and $\mathrm{i}^{\text {th }}$ pixel will be calculated, as. 


$$
d_{s}=\sqrt{\left.a_{j}+a_{i}\right)^{2}}+\left(b_{j}-b_{i}\right)^{2}
$$

With the normalized intensity of gray values $(\mathrm{N})$ of the $j^{\text {th }}\left(N_{j}\right)$ and $i^{\text {th }}\left(N_{i}\right)$ pixels, the intensity distance $\left(d_{c}\right)$ between the $j^{\text {th }}$ and $i^{\text {th }}$ pixels will follow (3).

$$
d_{c}=\sqrt{\left(N_{j}-N_{i}\right)^{2}}
$$

$\mathrm{S}$ is the Parmenter of local clustering $M$, the image numbers, and $k$ superpixels number is calculated by (4).

$$
S=\sqrt{M / K}
$$

If the compactness coefficient is $\mathrm{p}$ and initial superpixel to grid size is $S$ parameter, $p$ balance between $d_{s}$, and $d_{c}$ then the overall distance $D$ is calculated as (5).

$$
D=\sqrt{d_{s}^{2}+\left(\frac{d_{s}}{S}\right)} p^{2}
$$

While application confirms that both the space distances and intensity are within the same range and obtain an optimum compactness coefficient, the CT image intensities used in (5) are normalized to $[0,1]$. We generated $(\mathrm{K})$ to select the number of pixels such as 100,300, and 600 superpixels. And then, we construct a region adjacency graph (RAG). A sample pre-processed JPG image of a CT scan with grade II hemorrhage Figure 8, and super-pixelized image Figure 8(a) with compactness factor $\mathrm{p}$ as 0.1 , and grid size (S) as $10, K=100$, the Figure 8 (b) using factor $p$ as 0.2 and grid size (S) as $20, K=300$. Figure 8 (c) using factor $\mathrm{P}$ as $0.3, \mathrm{~K}=600$, is presented. We also noted that a greater value of the compactness coefficient creates more flexible boundaries, and a smaller value in more compact segments.

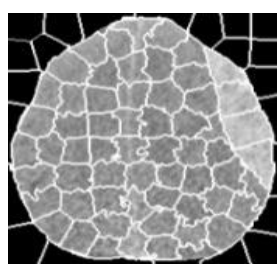

(a)

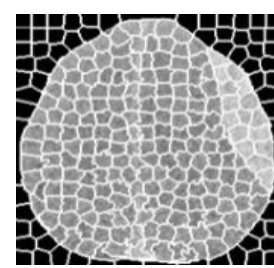

(b)

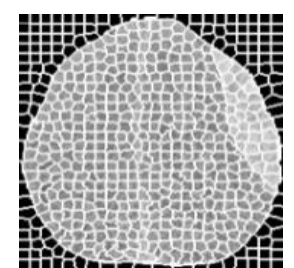

(c)

Figure 8. Segmentation output with different sizes of superpixels K: (a) the output of superpixels K=100, (b) the output of superpixels $K=300$, (c) the output of superpixels $K=600$.

\subsection{Edge detection}

In the proposed technique, we adopted the Canny edge detectors multi-stage algorithm to detect the abrupt functional changes in the average brain intensity. It identified the contour of the region of interest (ROI) [12], which is highly beneficial to find the brain hemorrhage position. The similarity between soft tissue edema and hemorrhages, these regions may be misdiagnosed as hemorrhage. We need to remove it by using an edge detection operator. To reduce the noise of data by Gaussian using the filter kernel of size $a \times a$ shown as (6), where $\sigma$ is the standard deviation.

$$
G(i, j)=\frac{1}{2 \pi \sigma^{2}} \exp \left(\frac{-(i-(a))^{2}+(j-(a))^{2}}{2 \sigma^{2}}\right) ; 1 \leq i, j \leq(a)
$$

The image is defined in the (7) and (8) is used to expand the segments smoothly. We calculated the low and high threshold by (7) and (8). It's more sophisticated and has a comparatively longer runtime and occasionally has false edges and a threshold is also necessary to identify and detecting edges.

$$
t_{\text {low }}=\max (0 \cdot(1.0-\text { sigma }) \times \text { median }(\text { image }))
$$




$$
t_{\text {high }}=\min (255,(1.0+\operatorname{sigma}) \times \operatorname{median}(\operatorname{image}))
$$

In addition to region growing methods are utilized for image segmentation in the next step for our technique. The watershed transforms which is a region growing method is based on three dimensions with image vision with $(x, y)$ coordinates on $x$ and $y$ axes and intensity on the $z$ axis. The comparative intensity values for high and low depend on grayscale darkness or brightness. The fact that watershed segmentation always produces closed contours is one of its benefits. Edge detection techniques are straightforward; however, it does not guarantee closed contours and are sensitive to threshold. Close contours provide the advantage of locating a seed pixel for each region, which then extends that region with its 8 -neighbors. The algorithm next checks for 8-connected neighbors to see if the pixels fit in this area or not (that is, if they are not members of other regions and are not edge pixels), then the pixels can be a part of this area. The connected components with connectivity $=8$ are adopted to find the largest contour conditioned with the size of contour area less than 5,000 pixels as shown in Figure 9. Figure 9(a) shows Edge detection using canny algorithm. Figure 9(b) displays output image after closing operation. The contours are labelled into different color by using connected component method as shown in Figure 9(c). The largest contour is then selected as presented in Figure 9(d).

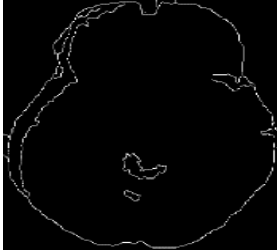

(a)

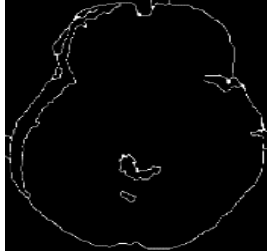

(b)

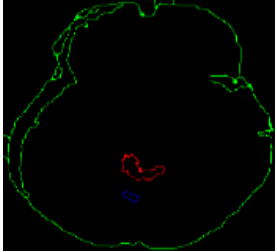

(c)

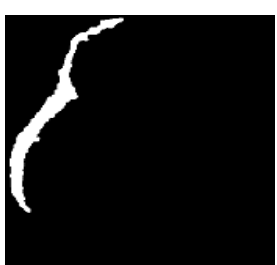

(d)

Figure 9. Different steps of morphological operation different shapes of hemorrhage: (a) edge detection using a canny algorithm, (b) closing operation, (c) find different contours, and (d) find the large contour

\subsection{Region growing}

The final step adopted in our approach is region growing [13]. We applied the automated seed point technique in region growing operations until no more pixels could be added to the region to eliminate the spurious regions and false boundaries while merging the hemorrhage sections. Select only the contours with the region $\mathrm{R}>10$ (remove all small contours) for the region growing method. Region growing with Threshold=18 with seed points above 8/10 heights of image. Further, 3D flood-based watershed transformation was applied for analyzing the intensity of brain hemorrhage. The explanatory shapes of the original figure, thresholding, and region growing were provided in Figure 10. Figure 10(a) demonstrates the original output after filtered by the specific range of HU. The noises of contour output are then removed by thresholding as illustrated in Figure 10(b). The region growing method is applied for the result as shown in Figure 10(c).

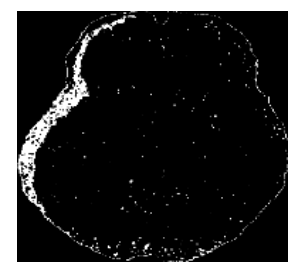

(a)

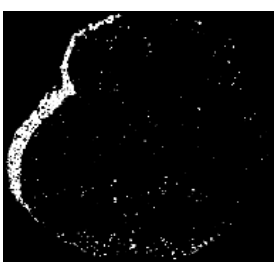

(b)

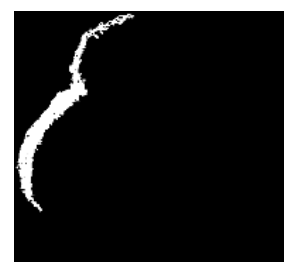

(c)

Figure 10. The output images during performing region growing approach: (a) image with noise, (b) image with less noise, and (c) image after region growing

\subsection{Dilation}

Dilation is based on the brain image's Morphology technique for free form object modeling the binary image. It represents the shape by extracting the object data from Figure 11(a) the result of the region growing, and Figure 11(b) shows the binary image after applying morphological filter size $9 \times 9$ to fill the 
hold. We have actualized the Dilation operations utilizing a fast-circular convolution method [14]. In the fastcircular convolution technique only defined, the kernel is padded up to the size of the output image for dilation. While the kernel is significantly smaller than the image, as is the case for most useful morphological image processing functions, the above circular convolution becomes inefficient due to the wasteful zeropadding involved. Dilation is increasing the pixels to fill the gap between pixels by fitting the injury's size. Erosion is decreeing the pixels outside the object of the image. Some regions of the image slice color look black. Another part of the region slice may look bright in comprehensive thresholding because of intensity non-similarity over the scene. The morphological closing operation to connecting the line neighbor with the kernel $3 \times 3$.
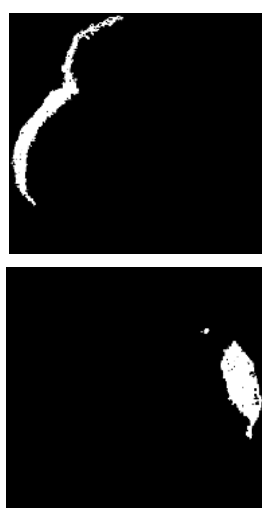

(a)
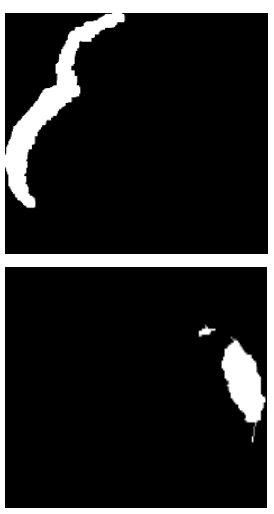

(b)

Figure 11. The result of dilation: (a) result of the region growth and (b) the large contour.

\subsection{Hybrid method}

In terms of the segmentation technique, we use the dice similarity coefficient (DSC) and, Jaccard similarity index (JSI) as the hybrid method to evaluate the performance. The high accuracy by combining dilation and edge detection for brain hemorrhage segmentation after removing noises with various filtering techniques and then covert a new image comes from the hybrid method with high performance.

$$
S=X \otimes Y
$$

Where, $X$ represents the SLIC result and $Y$ the output of the region growing method. Figure 12 shows the hybrid method result. Firstly, the edge detection method is shown in Figure 12(a), while the region growing method using dilation result is presented in Figure 12(b). Finally, high-performance results are achieved using a hybrid approach as shown in Figure 12(c).
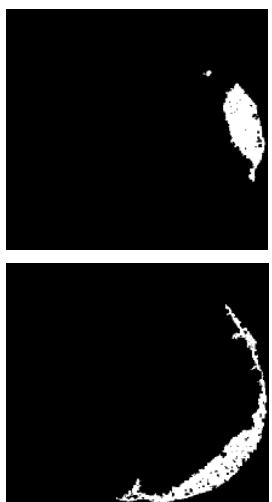

(a)
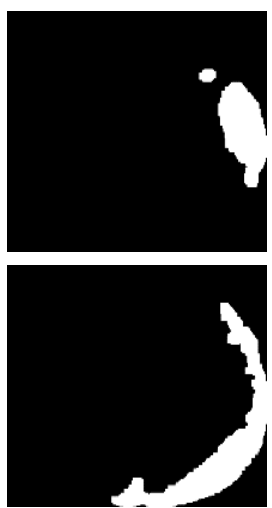

(b)
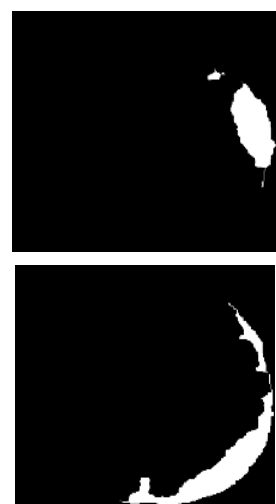

(c)

Figure 12. The hybrid method result, (a) edge detection, (b) dilation result and (c) exact effect for the combine 
The dice similarity coefficient (DSC), $M$ which stands for Segmentation, can be seen in (10) and is superior to overall pixel accuracy since it considers all false positive and missing values in each class. DSC is also thought to be superior because it defines the accuracy of the segmentation boundaries identified as well as the number of pixels correctly labeled.

$$
D S C=\frac{2\left|M_{I C H} \cap M_{I \overparen{C H}}\right|}{\left|M_{I C H}\right|+\left|M_{I C H}\right|}=\frac{2 \times T P}{2 \times T P+F N}
$$

The JSI also identified as intersection-over-union, $I o U$ is described as the ratio of the region of intersection between the ground truth $\left(M_{I \overparen{C H}}\right)$ and predicted segmentations $\left(M_{I C H}\right)$ to the region of union between the ground truth and predicted segmentations [15].

$$
J S I=\frac{M_{I C H} \cap M_{I \overparen{C H}}}{M_{I C H} \cup M_{I \overparen{C H}}}=\frac{T P}{T P+F P+F N}
$$

Where, $M$ the segmentation stands. JSI is like being monotonous in one additional or positively linked as DSC demonstrated in the previous equations. The difference between JSI the following and DSC the apparent one is that JSI the instances of wrong outcomes will be penalized more than the DSC situation. Therefore, one of these two metrics might be utilized instead of using both measures to validate segmentation as shows.

$$
J S I=\frac{D S C}{2-D S C} \times D S C=\frac{2 \times J S I}{1+J S I}
$$

For each brain image region of a CT scan, the pixels as hemorrhage or non-hemorrhage are considered. We believe the red pixels as edge detection clusters for brain hemorrhage images by using semi$\mathrm{K}$-means clustering. For noise reduction, a $3 \times 3$ median filter is applied to the image. The edge detection shape and segmentation region by using free-form object model are illustrated in Figure 13(a) and Figure 13(b), consecutively. The free-form object model is used to guarantee smoothness in the image while also ensuring no boundary information is missing [16].

Further, we adopted the multi-stage algorithm of the contour boundary detector to detect the abrupt functional changes in the normal brain's intensity. It identified the contour of the region of interest (ROI), which is highly beneficial to find the brain hemorrhage position [17]. The samples of three features and four features to cluster boundary region through contrast region pixels of the threshold process are improved in Figure 14(a) and Figure 14(b), respectively.
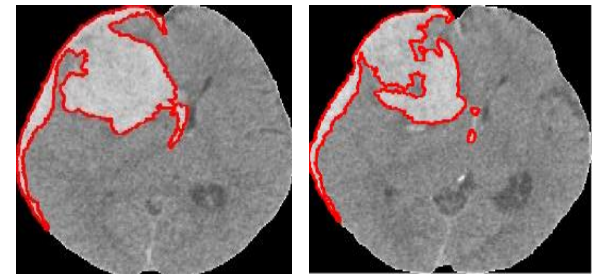

(a)

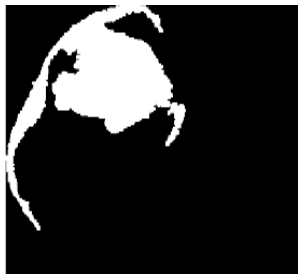

(b)

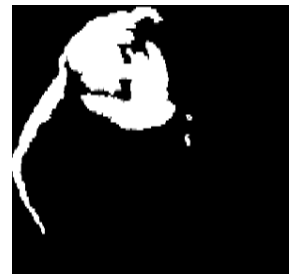

)

Figure 13. The region hemorrhage segmentation: (a) edge detection shape and (b) segmentation region

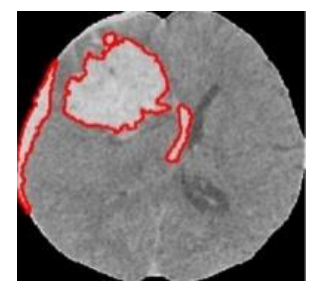

(a)

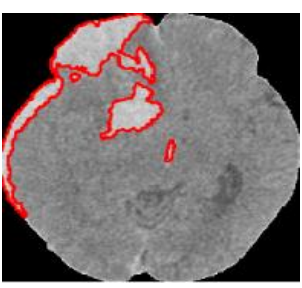

(b)

Figure 14. Different slices shapes of bleeding: (a) three features on shape and (b) four various features on shape 


\section{COLLECTION DATA}

This retrospective study required the dataset from Maharaj Nakorn Chiang Mai Hospital, Thailand, below specialist doctors. Standard digital imaging and communications in medicine (DICOM) axial slices, we collected a dataset consisting of 3D computed tomography (CT) brain Scan of patients involved more than 6520 slices labeled (intracerebral hemorrhage (ICH), epidural hematoma (EDH), subdural hematoma (SDH), midline shift (MLS), intraventricular hemorrhage (IVH)) and standard. Convert DICOM to JPEG Images. In this study, the research evaluates our proposed brain hemorrhages to evaluate different classifier mages set consisting of more than 5000 slices. Slice thickness was constant in most datasets use 300 patients' datasets had a variable thickness of slices between male and female patients between 25 and 61 years. The slice thickness size chosen $1.5 \mathrm{~mm}$ for each thickness has been 200-500 slices. 2D, the selected slices are composing a sample 3D CT brain scan as an illustration. Convert Hounsfield units of CT numbers (from -1000 to +1000 ) to Grayscale ( 0 to 255 ) by setting window width $=150$, window level $=30$, as indicated by doctor diagnosis

\section{EXPERIMENTAL RESULTS AND DISCUSSION}

For segmentation hemorrhage, the high accuracy of the free-form object model for clustering injury hemorrhage pixels is achieved. The proposed approach significant advantage is to attain high accuracy in clustering and a high $F_{1}$-Score with low computational power [18]. The superpixels generated through the SLIC [19] technique perform well in extracting brain hemorrhage complex texture using a different segmented number. Further, superpixels' spectral clustering using global Eigen decomposition achieved high accuracy in segmenting the regions and sophisticated brain hemorrhage features. Hence. The steps used and the outcomes are illustrated in Figure 15. Figure 15(a) shows the SLIC, output with superpixels K equals to 600. The regions with same pixel values are then merged as illustrated in Figure 15(b). The thresholding is then applied to the output of region merging as presented in Figure 15(c). Figure 15(d). The demonstrates the final segmentation output of the hemorrhage region. The proposed method outperforms conventional clustering methods in segmenting CT brain scans. The proposed approach also overcomes the dense similarity construction of spectral clustering after applying the proposed method, the free-form object model.
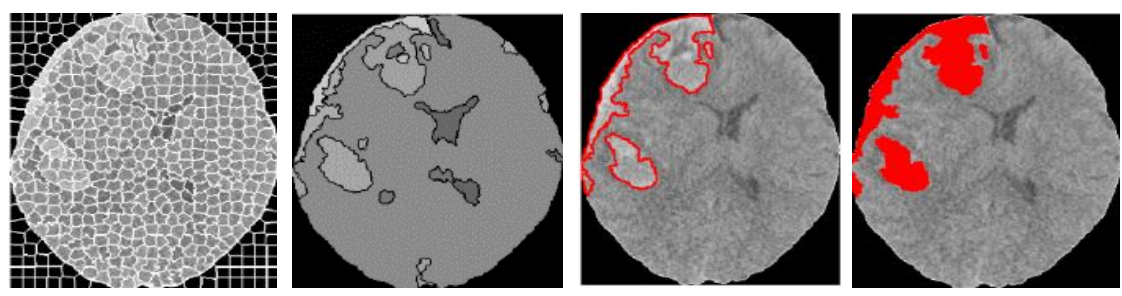

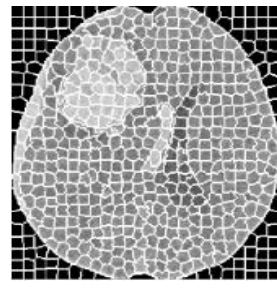

(a)

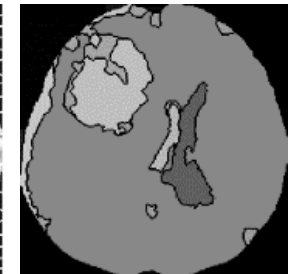

(b)

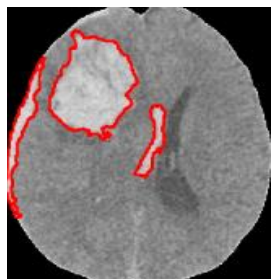

(c)

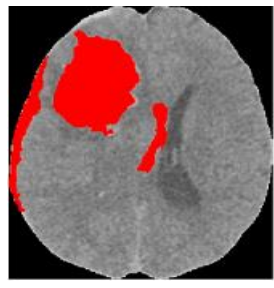

(d)

Figure 15. Different steps of our methods: (a) SLIC, (b) region merging, (c) thresholding and edge detection, and (d) segmentation output.

\section{PERFORMANCE MEASURES}

The segmentation result needs the right superpixel approach to enhance the segmentation method's performance. In this work, we compared our work with baseline methods in different metrics. Dice coefficient runs from 0 to 1 , where 1 stand for total overlap. In addition, the subsequent similarity measure is associated to the dice coefficient, by users based on the $512 \times 512$ mask of the CT slices resulted [20]. By using the ground truth segmentation from the ICH UNet dataset, we can calculate the JSI and t DSCof the ICH segmentation and high sensitivity in detecting the ICH regions to be considered as the following Table 1. 
Table 1. The comparison three methods UNet, projection profile and our method by using JSI and DSC

\begin{tabular}{ccc}
\hline Methods & JSI & DSC \\
\hline Projection Profile [21] & 0.66 & 0.21 \\
ICH UNet [22] & 0.24 & 0.33 \\
Our Method & 0.45 & 0.46 \\
\hline
\end{tabular}

A comparison results of the ICH UNet dataset, including 318 images with hemorrhage and 340 images without hemorrhage by testing on intracerebral hemorrhage the accuracy achieved to $82.37 \%$ shown in Table 2. We are using three methods for ICH UNet datasets. Statistical measures such as the accuracy, specificity, and $F_{1}$-score of three methods for one feature intracranial hemorrhage. Intracranial hemorrhage is analyzing CTscans by radiologists to identify ICH and focus its regions. To segment the region of the ICH in a fully automated manner. Therefore, we are using our dataset to apply for two methods, include projection profile and ICH UNet is considered to achieve high accuracy as indicated in Table 3, by used different parameter statistical measures.

Table 2. Three methods for ICH hemorrhage using physionet dataset

\begin{tabular}{ccccc}
\hline Methods & Sensitivity (\%) & Specificity (\%) & F1-Score (\%) & Accuracy (\%) \\
\hline Projection Profile [21] & 77.43 & 79.60 & 80.05 & 78.53 \\
ICH UNet [22] & 12.05 & 95.64 & 21.42 & 20.22 \\
Our Method & 78.93 & 85.59 & 81.22 & 82.37 \\
\hline
\end{tabular}

Table 3. The comparison of three methods for ICH hemorrhage using our dataset

\begin{tabular}{ccccc}
\hline Methods & Sensitivity (\%) & Specificity (\%) & F1-Score (\%) & Accuracy (\%) \\
\hline Projection Profile [21] & 69.44 & 91.85 & 67.30 & 87.77 \\
ICH UNet [22] & 87.32 & 82.82 & 45.59 & 74.71 \\
Our Method & 94.48 & 87.66 & 77.82 & 94.84 \\
\hline
\end{tabular}

TNR: Also known as specificity or Precision, measures the rate of true negatives concerning the number of real negative cases follow as (13) [23].

$$
\begin{aligned}
& T N R=\frac{T N}{T N+F P} \times 100 \% \\
& T P R=\frac{T P}{T P+F N} \times 100 \% \\
& F_{1}=\frac{2 T P}{2 T P+F P+F N} \times 100 \% \\
& A C C=\frac{T P+T N}{T P+T N+F P+F N} \times 100 \%
\end{aligned}
$$

Where TP is true positive (positive issue of correctly segmented) TN is true negative (Negative effect of appropriately segmented), FP is false positive (incorrectly segmented positive issue). FN is false negative (negative point of incorrectly segmented). To evaluate the three features of brain hemorrhage such as EDH, $\mathrm{SDH}$, and ICH segmented by different steps of slices such as 40 to 81 slices and varying range of $\mathrm{K}=600$, the performance of our method accurate and shown the average for their hemorrhage steps shown on Table 4. The accuracy measures used to evaluate the performance of the proposed method giving the best results when comparing with other methods show in Table 5.

Table 4. $\mathrm{K}=600$ is start from slice 40

\begin{tabular}{cccccc}
\hline & \multicolumn{2}{c}{ K=600 } & \multicolumn{3}{c}{ Start from Slice 40 } \\
& PPV & TPR & F1 & TNP & ACC \\
\hline EDH & 97.08 & 97.24 & 97.16 & 88.89 & 95.50 \\
SDH & 97.69 & 97.53 & 97.61 & 89.55 & 96.09 \\
ICH & 97.19 & 96.69 & 96.94 & 90.09 & 93.07 \\
Average & 97.32 & 97.15 & 97.23 & 89.51 & 94.88 \\
\hline
\end{tabular}


Table 5. Retrieval accuracy of the hemorrhage by using greater papers

\begin{tabular}{ccccc}
\hline Method & EDH & ICH & SDH & Average \\
\hline$[24]$ & 86.3 & - & 84.3 & 85.3 \\
{$[25]$} & 72.0 & 87.0 & 84.0 & 81 \\
{$[26]$} & 62.3 & 82.2 & 54.1 & 66.2 \\
{$[27]$} & 48.9 & 88.4 & 71.8 & 69.7 \\
{$[28]$} & 72.7 & 96.6 & 89.1 & 86.1 \\
{$[29]$} & 48.9 & 88.4 & 71.8 & 69.7 \\
Our Method & 97.06 & 99.81 & 96.61 & 97.16 \\
\hline
\end{tabular}

\section{CONCLUSION AND FUTURE WORK}

A hybrid hemorrhage segmentation and measurement technique are presented in this paper. Traumatic brain injuries are a significant process in assisting physicians in diagnosis the injury for medical image processing. The segmentation method is based on the superpixel technique for segmenting hemorrhage in brain scans by used a novel freeform object model. The images are computing SLIC to object boundaries in the initial step. The pre-processing techniques for noise reduction and removing unwanted regions from CT scan brain images. To detect the feature of hemorrhage is obtains as red marked. Preliminary results of using images from our dataset illustrated that the current approach of boundary edge detection and brain hemorrhage segmentation achieved $94.84 \%$ accuracy. Finally, this method in the clinical experimental setting will decrease clinicians' valuable time required to diagnose due to the technique that can provide hemorrhage segmentation. For future work, we will involve more features of TBI by apply AI field specially machine learning algorithms for the classification step to be more benefit for doctor system diagnoses.

\section{ACKNOWLEDGMENTS}

The researchers thank the international college of digital innovation Chiang Mai University for giving to us the opportunity and the Faculty of Medicine's support through the research fund, grant no. 143-2562 to help our group to collect the datasets of hemorrhage CT scan images.

\section{REFERENCES}

[1] B. Peng, L. Zhang, and D. Zhang, "A survey of graph theoretical approaches to image segmentation," Pattern Recognition, vol. 46, no. 3, pp. 1020-1038, Mar. 2013, doi: 10.1016/j.patcog.2012.09.015.

[2] P. Ebenezer, H. Nunoo-Mensah, R. Junior, and J. Raissa, "Brain tumor segmentation using SLIC superpixels and optimized thresholding algorithm," International Journal of Computer Applications, vol. 181, no. 20, pp. 1-5, Oct. 2018, doi: 10.5120/ijca2018917915.

[3] G. Litjens et al., "A survey on deep learning in medical image analysis," Medical Image Analysis, vol. 42, pp. 60-88, Dec. 2017, doi: 10.1016/j.media.2017.07.005.

[4] S. Zhang, Z. You, and X. Wu, "Plant disease leaf image segmentation based on superpixel clustering and EM algorithm," Neural Computing and Applications, vol. 31, no. S2, pp. 1225-1232, Feb. 2019, doi: 10.1007/s00521-017-3067-8.

[5] H. E. Tasli, C. Cigla, and A. A. Alatan, "Convexity constrained efficient superpixel and supervoxel extraction," Signal Processing: Image Communication, vol. 33, pp. 71-85, Apr. 2015, doi: 10.1016/j.image.2015.02.005.

[6] J. Cong, B. Wei, Y. Yin, X. Xi, and Y. Zheng, "Performance evaluation of simple linear iterative clustering algorithm on medical image processing," Bio-Medical Materials and Engineering, vol. 24, no. 6, pp. 3231-3238, 2014, doi: 10.3233/BME-141145.

[7] M. D. Hssayeni, M. S. Croock, A. D. Salman, H. F. Al-khafaji, Z. A. Yahya, and B. Ghoraani, "Intracranial hemorrhage segmentation using a deep convolutional model," Data, vol. 5, no. 1, Art. no. 14, Feb. 2020, doi: 10.3390/data5010014.

[8] W.-D. Zech, C. Jackowski, Y. Buetikofer, and L. Kara, "Characterization and differentiation of body fluids, putrefaction fluid, and blood using Hounsfield unit in postmortem CT," International Journal of Legal Medicine, vol. 128, no. 5, pp. 795-802, Sep. 2014, doi: 10.1007/s00414-014-1030-0.

[9] S. Csaholczi, D. Iclănzan, L. Kovács, and L. Szilágyi, "Brain tumor segmentation from multi-spectral MR image data using random forest classifier," in Lecture Notes in Computer Science, Springer International Publishing, pp. 174-184, 2020.

[10] M. R. Al-Hadidi, B. AlSaaidah, and M. Al-Gawagzeh, "Glioblastomas brain tumour segmentation based on convolutional neural networks," International Journal of Electrical and Computer Engineering (IJECE), vol. 10, no. 5, pp. 4738-4744, Oct. 2020, doi: 10.11591/ijece.v10i5.pp4738-4744.

[11] A. Khosravanian, M. Rahmanimanesh, P. Keshavarzi, and S. Mozaffari, "Fast level set method for glioma brain tumor segmentation based on Superpixel fuzzy clustering and lattice Boltzmann method," Computer Methods and Programs in Biomedicine, vol. 198, Art. no. 105809, Jan. 2021, doi: 10.1016/j.cmpb.2020.105809.

[12] F. Hossain, M. Asaduzzaman, M. Abu, and M. Armanur, "Dynamic thresholding based adaptive canny edge detection," International Journal of Computer Applications, vol. 135, no. 4, pp. 37-41, Feb. 2016, doi: 10.5120/ijca2016908337.

[13] A. Aslam, E. Khan, and M. M. S. Beg, "Improved edge detection algorithm for brain tumor segmentation," Procedia Computer Science, vol. 58, pp. 430-437, 2015, doi: 10.1016/j.procs.2015.08.057.

[14] R. B. Holmes, I. S. Negus, S. J. Wiltshire, G. C. Thorne, and P. Young, "Creation of an anthropomorphic CT head phantom for verification of image segmentation," Medical Physics, vol. 47, no. 6, pp. 2380-2391, Jun. 2020, doi: 10.1002/mp.14127.

[15] I. Rizwan I Haque and J. Neubert, "Deep learning approaches to biomedical image segmentation," Informatics in Medicine Unlocked, vol. 18, Art. no. 100297, 2020, doi: 10.1016/j.imu.2020.100297.

[16] H. Chen, S. Khan, B. Kou, S. Nazir, W. Liu, and A. Hussain, "A smart machine learning model for the detection of brain hemorrhage diagnosis based internet of things in smart cities," Complexity, vol. 2020, pp. 1-10, Sep. 2020, doi: $10.1155 / 2020 / 3047869$. 
[17] G. Wang, W. Li, M. Aertsen, J. Deprest, S. Ourselin, and T. Vercauteren, "Aleatoric uncertainty estimation with test-time augmentation for medical image segmentation with convolutional neural networks," Neurocomputing, vol. 338, pp. 34-45, Apr. 2019, doi: 10.1016/j.neucom.2019.01.103.

[18] M. D. Hssayeni, M. S. Croock, A. D. Salman, H. F. Al-khafaji, Z. A. Yahya, and B. Ghoraani, "Intracranial hemorrhage segmentation using a deep convolutional model," Data, vol. 5, no. 1, Art. no. 14, Feb. 2020, doi: 10.3390/data5010014.

[19] Y. Wu, Z. Zhao, W. Wu, Y. Lin, and M. Wang, "Automatic glioma segmentation based on adaptive superpixel," BMC Medical Imaging, vol. 19, no. 1, p. 73, Dec. 2019, doi: 10.1186/s12880-019-0369-6.

[20] C. S. S. Anupama, M. Sivaram, E. L. Lydia, D. Gupta, and K. Shankar, "Synergic deep learning model-based automated detection and classification of brain intracranial hemorrhage images in wearable networks," Personal and Ubiquitous Computing, Nov. 2020, doi: 10.1007/s00779-020-01492-2.

[21] N. S. M. Noor, N. M. Saad, A. R. Abdullah, and N. M. Ali, "Automated segmentation and classification technique for brain stroke," International Journal of Electrical and Computer Engineering (IJECE), vol. 9, no. 3, pp. 1832-1841, Jun. 2019, doi: 10.11591/ijece.v9i3.pp1832-1841.

[22] Z. U. Rehman, S. S. Naqvi, T. M. Khan, M. A. Khan, and T. Bashir, "Fully automated multi-parametric brain tumour segmentation using superpixel based classification,” Expert Systems with Applications, vol. 118, pp. 598-613, Mar. 2019, doi: 10.1016/j.eswa.2018.10.040.

[23] Ahmad Yahya Dawod, J. Abdullah, and M. J. Alam, "A new method for hand segmentation using free-form skin color model," in 2010 3rd International Conference on Advanced Computer Theory and Engineering(ICACTE), Aug. 2010, pp. V2-562-V2-566, doi: 10.1109/ICACTE.2010.5579466.

[24] M. van Eijnatten, R. van Dijk, J. Dobbe, G. Streekstra, J. Koivisto, and J. Wolff, "CT image segmentation methods for bone used in medical additive manufacturing," Medical Engineering and Physics, vol. 51, pp. 6-16, Jan. 2018, doi: 10.1016/j.medengphy.2017.10.008.

[25] P. D. Chang et al., "Hybrid 3D/2D convolutional neural network for hemorrhage evaluation on head CT," American Journal of Neuroradiology, vol. 39, no. 9, pp. 1609-1616, Sep. 2018, doi: 10.3174/ajnr.A5742.

[26] H. Ye et al., "Precise diagnosis of intracranial hemorrhage and subtypes using a three-dimensional joint convolutional and recurrent neural network," European Radiology, vol. 29, no. 11, pp. 6191-6201, Nov. 2019, doi: 10.1007/s00330-019-06163-2

[27] G. Danilov et al., "Classification of intracranial hemorrhage subtypes using deep learning on CT scans," Studies in Health Technology and Informatics, vol. 272, pp. 370-373, 2020.

[28] M. Burduja, R. T. Ionescu, and N. Verga, "Accurate and efficient intracranial hemorrhage detection and subtype classification in 3D CT scans with convolutional and long short-term memory neural networks," Sensors, vol. 20, no. 19, Art. no. 5611, Oct. 2020, doi: $10.3390 / \mathrm{s} 20195611$.

[29] A. Sage and P. Badura, "Intracranial hemorrhage detection in head CT using double-branch convolutional neural network, support vector machine, and random forest," Applied Sciences, vol. 10, no. 21, Art. no. 7577, Oct. 2020, doi: 10.3390/app10217577.

\section{BIOGRAPHIES OF AUTHORS}

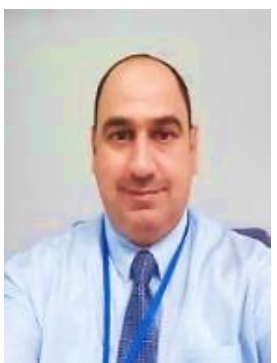

Ahmad Yahya Dawod (DD SC S P received his BSc from the Faculty of Computer Science at Al-Mustansiriya University, Iraq in 2006. He completes his Master degree from the Faculty of Computing and informatics at Multimedia University-MMU Cyberjaya, Malaysia in 2012. He completes his Ph.D. from the Faculty of information science and technology at National University of Malaysia in 2018. He is currently a lecturer at International College of Digital Innovation Chiang Mai University, Chiang Mai, Thailand. His research interests include Artificial Intelligent, Machine Learning, Pattern Recognition, computer vision, Image Processing, and Medical Image Analysis. Email: ahmadyahyadawod.a@cmu.ac.th.
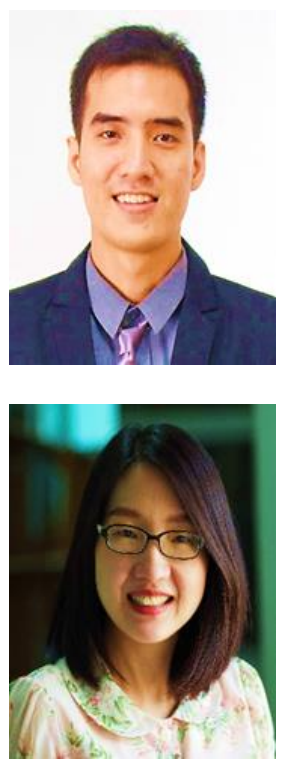

Aniwat Phaphuangwittayakul (iD) 80 SC P received B.Eng. from the Faculty of Computer Engineering, Chiang Mai University, Thailand, in 2012, and Master of Science from Beijing Institute of Technology, Beijing, China, in 2017. He is currently a Lecturer in the International College of Digital Innovation, Chiang Mai University as well as a Ph.D. candidate in East China University of Science and Technology, China. His current research interests are Meta-Learning, Deep Generative Model, Computer Vision and Artificial Intelligence. Email:

Int J Elec \& Comp Eng, Vol. 12, No. 2, April 2022: 1437-1448
Salita Angkurawaranon (iD) $8 \mathrm{SC}$ P received her medical degree from Chiang Mai University in 2005 and finished her specialization in Radiology in 2009. She finished her fellowship is neuroradiology from Ramathibodi Hospital, Mahidol University in 2014. She is current an Assistant Professor in neuroimaging at the Department of Radiology, Faculty of Medicine, Chiang Mai University. Email: 